\title{
OPTimizing Irradiation through Molecular Assessment of Lymph node (OPTIMAL): a randomized open label trial
}

\author{
Manuel Algara López ${ }^{1 *} \mathbb{0}$, Elvira Rodríguez García², Inmaculada Beato Tortajada ${ }^{3}$, \\ Francisco José Martínez Arcelus ${ }^{4}$, Juan Salinas Ramos ${ }^{5}$, José Reyes Rodríguez garrido ${ }^{6}$, Xavier Sanz Latiesas ${ }^{7}$, \\ Ana Soler Rodríguez ${ }^{8}$, Germán Juan Rijo ${ }^{9}$ and Amanda Flaquer García ${ }^{10}$
}

\begin{abstract}
Background: Conservative surgery followed by breast and nodal irradiation is the standard loco-regional early breast cancer (BC) treatment for patients with four or more involved lymph nodes. However, the treatment strategy when fewer nodes are involved remains unclear, especially when lymphadenectomy has not been performed. Sensitive nodal status assessment molecular techniques as the One-Step Nucleic Acid Amplification (OSNA) assay can contribute to the definition and standardization of the treatment strategy. Therefore, the OPTIMAL study aims to demonstrate the feasibility of incidental irradiation of axillary nodes in patients with early-stage BC and limited involvement of the SLN.

Methods: BC patients who underwent conservative surgery and whose SLN total tumour load assessed with OSNA ranged between 250-15,000 copies/ML will be eligible. Patients will be randomized to receive irradiation on the breast, tumour bed, axillary and supraclavicular lymph node areas (intentional arm) or only on the breast and tumour bed (incidental arm). All areas, including the internal mammary chain, will be contoured. The mean, median, D5\% and D95\% doses received in all volumes will be calculated. The primary endpoint is the non-inferiority of the incidental irradiation of axillary nodes compared to the intentional irradiation in terms of 5 -year disease free survival. Secondary endpoints comprise the comparison of acute and chronic toxicity and loco-regional and distant disease recurrence rates.

Discussion: Standardizing the treatment and diagnosis of $B C$ patients with few nodes affected is crucial due to the lack of consensus. Hence, the quantitative score for the metastatic burden of SLN provided by OSNA can contribute by improving the discrimination of which BC patients with limited nodal involvement can benefit from incidental radiation as an adjuvant treatment strategy.
\end{abstract}

Trial registration: ClinicalTrial.gov, NCT02335957; https://clinicaltrials.gov/ct2/show/NCT02335957

Keywords: Breast cancer, Conserving surgery, OSNA, Nodal irradiation, Incidental dose

\footnotetext{
*Correspondence: malgara@parcdesalutmar.cat

${ }^{1}$ Radiation Oncology Department, Del Mar Hospital, Autonomous

University of Barcelona, Hospital del Mar Medical Research Institute, Passeig Maritim, 25, 08003 Barcelona, Spain

Full list of author information is available at the end of the article
}

\section{Background}

It has been shown that breast and nodal irradiation reduce mortality and disease recurrence in breast cancer (BC) patients [1]. Therefore, there is evidence supporting the use of adjuvant radiotherapy after conservative surgery. Since nodal irradiation increases survival rates, it is recommended in patients with four or more involved original author(s) and the source, provide a link to the Creative Commons licence, and indicate if changes were made. The images or other third party material in this article are included in the article's Creative Commons licence, unless indicated otherwise in a credit line to the material. If material is not included in the article's Creative Commons licence and your intended use is not permitted by statutory regulation or exceeds the permitted use, you will need to obtain permission directly from the copyright holder. To view a copy of this licence, visit http://creativecommons.org/licenses/by/4.0/. The Creative Commons Public Domain Dedication waiver (http://creativeco mmons.org/publicdomain/zero/1.0/) applies to the data made available in this article, unless otherwise stated in a credit line to the data. 
lymph nodes (LN) [2], as well as in patients with other types of locally advanced tumours with LN involvement [3]. A randomized clinical trial carried out by the British Columbia Cancer Agency, showed that the 20-year disease-free survival (DFS) of patients subjected to the combination of chemotherapy and radiation was 13\% higher than that of the patients who were treated with chemotherapy alone [4]. Similar results were achieved in the DBCG 82 b\&c trial showing a difference of $10 \%$ in the 15-year overall survival rate between the treatment combination and chemotherapy alone (39\% vs. $29 \%$, respectively) [5]. Furthermore, a study funded by the Canadian Cancer Society concluded that adjuvant radiation treatment of limited LN-positive patients $(0-3$ involved nodes) led to a decrease in disease recurrence [6]. However, there is no consensus on the recommendation of radiotherapy when the nodal involvement ranges between 1 and 3 LN [7].

Furthermore, this situation became even more unclear since Giuliano et al. demonstrated no significant benefit in loco-regional control with completion of axillary lymph node dissection (ALND) in comparison to no ALND in patients with 1-2 involved LN [8,9]. On the other hand, AMAROS [10] and OTOASOR [11] trials demonstrated that nodal irradiation should be regarded as the recommended treatment for patients with few involved LN, instead of ALND. In line with their results, the Canadian trial NCIC-CTG MA20 [6] showed that local irradiation combined with regional irradiation improved the DFS as well as the loco-regional and distant control of the disease in high-risk patients with negative and with positive LN, mostly with 1 to 3 involved LN, while Poortmans et al. [12] demonstrated that the irradiation of the regional nodes in patients with limited axillary disease results in an increase of the DFS. Darby et al. [13] reported that, after breast-conserving surgery, the application of radiotherapy to the breast reduced mortality and the disease recurrence by half. Finally, a systematic review, which included more than 20,000 patients from 45 studies, concluded that breast irradiation reduced the loco-regional relapse even in patients without LN involvement [14]. Later on, the meta-analysis conducted by Budach et al. $[15,16]$ concluded that additional regional radiation to the internal mammary and medial supraclavicular LN improved overall and diseasefree survival rates in stage I-III BC patients.

The uncertainty about the proper radiation therapy entails the need for a method that standardizes the choice of treatment and complements the limited diagnostic information [17-19], such as nodal involvement assessment. The OSNA (One-Step Nucleic Acid Amplification) assay provides a quantitative value of the metastatic burden of the sentinel lymph node (SLN) by measuring the
mRNA expression of the tumour marker cytokeratin 19 (CK19) [20]. The OSNA assay not only provides automated and complete intraoperative analyses of the SLN but also standardized and reliable results for SLN metastatic status. The total tumour load (TTL), defined as the sum of the CK19 mRNA copies from all positive SLNs of the patient, entails a quantitative score that integrates both the metastatic burden and the number of involved SLN. The TTL score was proved to be an independent predictor factor of the axillary nodal status, where only $14.7 \%$ of patients with TTL beneath 15.000 copies $/ \mu \mathrm{l}$ had other positive non-SLN [21]. Recently, the PLUTTO study results also proved its impact on the prognosis of $\mathrm{BC}$ patients [22].

Therefore, the OPTIMAL study (OPTimizing Irradiation through Molecular Assessment of Lymph node) aims to demonstrate the non-inferiority of incidental irradiation of axillary nodes in comparison to intentional irradiation in terms of the 5-year DFS of patients with early-stage BC and limited involvement of the SLN according to the OSNA quantitative score.

\section{Methods/design Study design}

The OPTIMAL study is an open-label multicentre and international trial (NCT02335957) conducted in over 40 sites in Spain, Portugal, and Italy. Eligible patients will be randomized with a 1:1 allocation ratio to receive irradiation on breast, tumour bed, axillary and supraclavicular lymph node areas (intentional arm) or only on breast and tumour bed (incidental arm), as depicted in Fig. 1. Randomization by blocking within centres to minimize imbalance of treatments among centres will be performed using the online randomization software RANDI2 (www. dkfz.de; Heidelberg University) embedded in the electronic case record form (eCRF). All patients will have to provide their Informed Consent prior to the inclusion in the study.

\section{Eligibility criteria Inclusion criteria}

- Older than 18 years old

- Female invasive ductal breast cancer patients

- T1-T2 tumour stage

- Previous treatment with breast-conserving surgery without axillary lymphadenectomy

- OSNA assayed SLN with a TTL within the 25015,000 copies/ $\mu \mathrm{L}$ range

- Karnofsky Performance scale Index $\geq 70 \%$

- Written and signed Informed Consent 


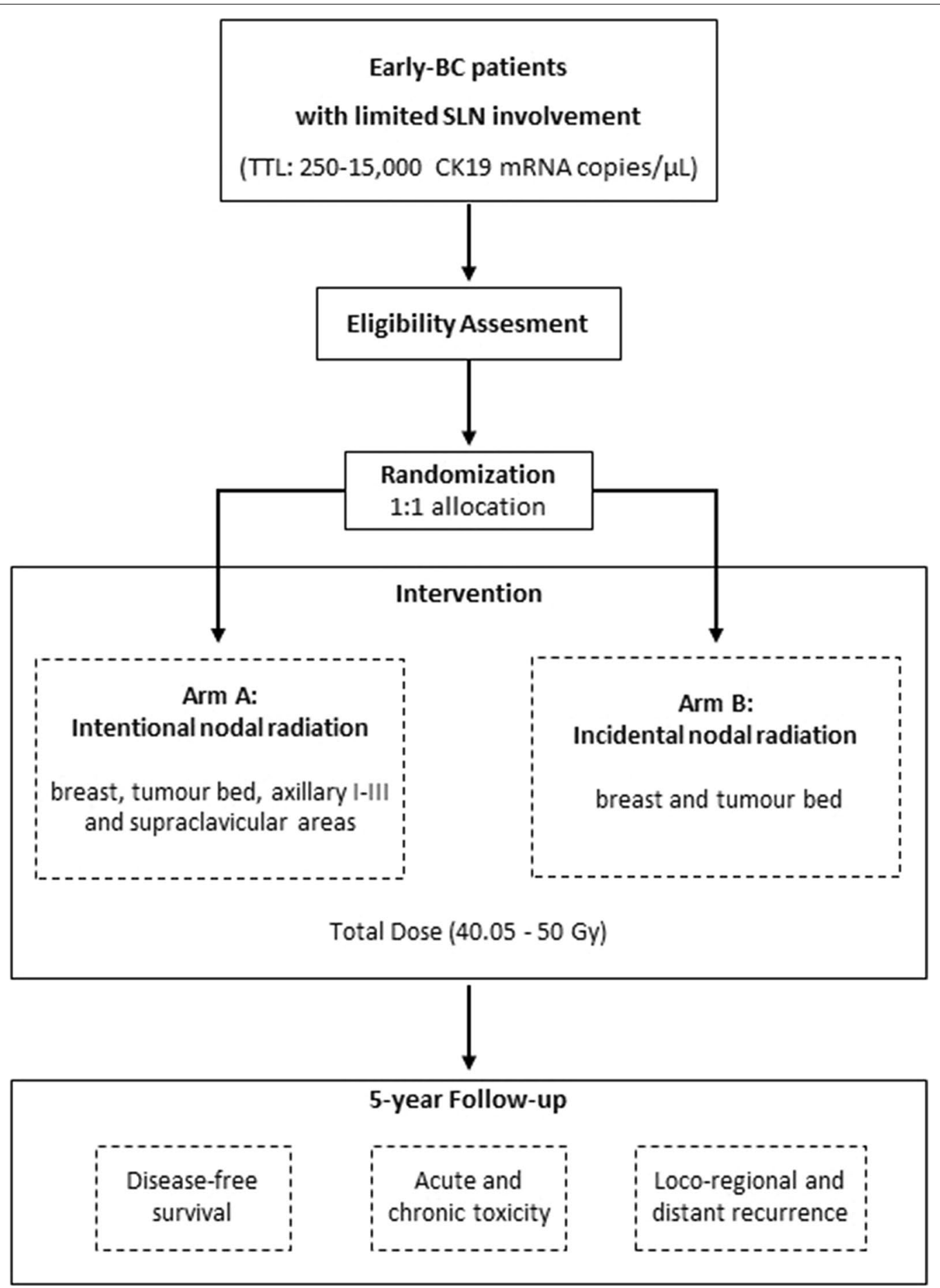

Fig. 1 OPTIMAL-I study design. BC breast cancer, CK19 cytokeratin 19, mRNA messenger ribonucleic acid, SLN sentinel lymph node, TTL total tumour load 


\section{Exclusion criteria}

- Invasive lobular carcinoma and other histologic subtypes

- Bilateral breast cancer

- Male breast cancer patients

- Patients who underwent a mastectomy or ipsilateral dissection of axillary LN

- Having received previous thoracic irradiation

- Systemic neoadjuvant therapy prior to surgery

- Contraindications to radiotherapy such as pregnancy or serious collagen disease.

- Other neoplasms and or any associated sever comorbidities that may interfere with the study

\section{Endpoints}

\section{Primary endpoint}

The primary endpoint is the non-inferiority of incidental irradiation of axillary nodes in contrast to intentional irradiation, in terms of the 5-year DFS of patients diagnosed with early-stage breast cancer with limited involvement of the SLN treated with breast-conservative surgery without axillary lymphadenectomy.

\section{Secondary endpoints}

The secondary endpoints established were the following:

- Loco-regional tumour recurrence in the two treatment arms within the 5-year follow-up period.

- Distant tumour recurrence in the two treatment arms within the 5-year follow-up period.

- Acute toxicity induced by either the incidental or intentional radiation treatment.

- Chronic toxicity induced by either the incidental or intentional radiation treatment.

- Total irradiation dose (Gy) received in axillary levels I-III, supraclavicular fossa, and internal mammary chain volumes.

\section{Radiation procedure Volume contouring}

All node areas from axillary levels I-III, the supraclavicular fossa and the internal mammary chain must be contoured in all patients regardless of the assigned arm following the guidelines of the Radiation Therapy Oncology Group [23]. The Clinical Target Volumes (CTV) will be delimited as stated below:

- Breast The CTV includes the whole breast's soft tissues ranging from $5 \mathrm{~mm}$ below the skin surface to the deep fascia, excluding the muscle and underlying ribs. The posterior margin should not extend beyond the deep fascia or the edges of the visible/ palpable breast in medial and lateral directions.

- Tumour bed The delineation of the tumour bed is only mandatory for patients who require a boost. It is strongly recommended to delimit the medial, lateral, superior, inferior, anterior and posterior margins of the surgical cavity at the time of the surgery, with clips or gold seeds. In the absence of implanted fiducial markers, the tumour bed may be localised if there is a well-defined seroma, considering visible changes in the computed tomography $(\mathrm{CT})$ or by means of support from previous mammograms or magnetic resonance imaging. This volume shall be defined by drawing around the implanted markers and changes in the surrounding tissue architecture.

- Supraclavicular In the incidental arm, nodes will be contoured as part of the procedure whereas, in the control group, this will be hidden so as not to influence the dosimetric planning. The limits will be cranial edge (thyroid cartilage); caudal edge (clavicle head); medial edge $(1 \mathrm{~cm}$ lateral to the lateral wall of the trachea excluding the thyroid); lateral edge (acromioclavicular joint); anterior edge (the sternocleidomastoid muscle and the clavicle); posterior edge (the trapezius muscle).

- Axilla level III In the incidental arm, nodes will be contoured as part of the procedure whereas, in the control group, this will be hidden to not influence the dosimetric planning. The limits will be the cranial edge ( $5 \mathrm{~mm}$ cranial to the axillary vessels); caudal edge ( $1 \mathrm{~cm}$ caudal to the axillary vessels); medial edge (the ribcage); lateral edge (the res major muscle); anterior edge (the pectoralis major muscle); posterior edge (chest wall and intercostal muscles).

- Axilla level II In the incidental arm, nodes will be contoured as part of the procedure whereas, in the control group, this will be hidden to not influence the dosimetric planning. The limits will be the cranial edge ( $5 \mathrm{~mm}$ cranial to the axillary vessels); caudal edge (caudal edge of pectoralis minor muscle); medial edge (the medial border of the pectoralis minor muscle); lateral edge (the lateral border of the pectoralis minor muscle); anterior edge (the anterior surface of the pectoralis minor muscle); posterior edge (chest wall and intercostal muscles).

- Axilla level I In the incidental arm, nodes will be contoured as part of the procedure whereas, in the control group, this will be hidden to not influence the dosimetric planning. The limits will be the cranial edge ( $1 \mathrm{~cm}$ below caput humeri); caudal edge (free edge of pectoralis major muscle); medial edge (the 
lateral border of the pectoralis minor muscle); lateral edge (the medial edge of the dorsolateral muscle); anterior edge (the plane defined by the anterior surface of the pectoralis major and minor lateral dorsal); posterior edge (the anterior surface of the subscapularis muscle).

- Internal mammary chain Nodes will be contoured in both arms and will be hidden to not influence the dosimetric planning. The limits will be the cranial edge (cranial edge of rib 1); caudal edge: (cranial edge of rib 5); medial edge (the edge of the sternum); lateral edge ( $5 \mathrm{~mm}$ lateral of internal mammary vessels or $2 \mathrm{~cm}$ from the edge of the sternum); anterior edge (dorsal surface of $\mathrm{m}$. pectoralis major, dorsal surface of the sternum); posterior edge (pleura).

- Organs at risk The delineation of ipsilateral lung and heart is mandatory for the calculation of dosevolume histograms. The ipsilateral lung must be outlined as a single structure, and care should be taken not to include any airways; the CT must cover the entire lung volume. The heart must be outlined as a single structure to the extent of the pericardial sac; the major blood vessels (superior and inferior) are excluded and the superior extent may be simplified by identifying the vessels superior to the heart. Contralateral breast, contralateral lung, oesophagus, caput humeri, thyroid and spinal cord may be delineated according to institutional protocols.

Enlarging all volumes by adding a $5-10 \mathrm{~mm}$ margin in all directions to create the planning treatment volume (PTV) is recommended.

\section{Treatment planning}

The treatment planning must be carried out on a 3D system with tissue heterogeneity correction and matrix resolution of $2.5 \mathrm{~mm}$. Techniques in the supine position are allowed and the prone position is not allowed.

- Breast Tangent beam pair arrangement to encompass the whole breast is recommended, intending to minimize the total dose in the ipsilateral lung and heart. The treatment plan can be optimized with any dose compensation system, (virtual edges, mechanical wedges, automatic wedges, field-in-field IMRT and steep and shoot or sliding windows IMRT). The treatment will be performed with photons of 6 MV-15 MV. The isocenter will be located within the breast PTV in the experimental group and may be located outside the PTV if the single isocenter technique is being used in the control group. To minimize irradiated ipsilateral lung and heart volumes, collima- tor rotation and shield with multileaf collimator is allowed.

- Tumour bed Photons or electrons are allowed. Minitangential photon fields or single electron field (also mixed energies) can be utilised. The use of boluses can be considered if needed. Simultaneous integrated boost brachytherapy or intraoperative irradiation is also allowed if the dose contribution to the nodal areas can be calculated. The bolus can be used. The dose and fractionation choice is left at the discretion of the treating physician.

- Node areas In the control group, the treatment planning must be optimized to ensure that the nodal areas receive the prescribed dose (except for the internal mammary chain), minimizing the dose at the organs at risk. High tangent, AP/PA, conformational 3D and IMRT techniques are allowed. In the experimental group, treatment planning for nodal areas is not performed. The internal mammary chain will not be irradiated intentionally in any case.

An unplanned gap of up 3 days is acceptable. Longer non-planned interruptions should be compensated by hyperfractionation of the daily normal dose.

\section{Dose prescription}

All doses prescribed will follow the International Commission on Radiation Units and Measurements guidelines. A minimum of $95 \%$ of the volume must receive at least $95 \%$ of the prescribed dose. Less than $5 \%$ of the volume may receive a dose of $105 \%$ and less than $2 \%$ should receive a dose of $107 \%$, with a maximum overall dose of $110 \%$. The dose in the breast must be 50.0 Gy by 25 fractions of $2.0 \mathrm{~Gy}$ in 5 weeks or through hypofractionated schedules as 40.05 Gy in 15 fractions of 2.67 Gy for 3 weeks. In the tumour bed, the schedule and total dose are left to the centre's criteria. In node areas, the intentional irradiation to the breast and tumour bed will be calculated. Limiting doses for the organs at risk must be: (1) ipsilateral lung: V20 less than 25\%; and (2) heart: V20 less than $10 \%$ and V40 less than $5 \%$.

\section{Radiotherapy verification}

Verification methods will be conducted in both arms. Treatment verification is required at the first treatment fraction and allowed on the three first fractions. The verification must be performed using electronic portal images of the treatment beam; either with $\mathrm{MV}$ or $\mathrm{kV}$. Orthogonal images or cone-beam images can be used on the verification of the isocentre. Weekly control will be performed, and systematic daily control is also allowed. 
Table 1 Schedule of visits and assessments (dots) that will be performed during the 5-year follow-up period

\begin{tabular}{|c|c|c|c|c|c|c|c|c|c|c|c|c|c|}
\hline Post-intervention (year) & 1 & & & & & 2 & & 3 & & 4 & & 5 & \\
\hline Post-intervention (month) & 1 & 3 & 6 & 9 & 12 & 18 & 24 & 30 & 36 & 42 & 48 & 54 & 60 \\
\hline \multicolumn{14}{|l|}{ Acute toxicity } \\
\hline \multicolumn{14}{|l|}{ Physical exam } \\
\hline \multicolumn{14}{|l|}{ Image evaluation of local recurrence } \\
\hline \multicolumn{14}{|l|}{ Survival and disease recurrence } \\
\hline Chronic toxicity & \multicolumn{13}{|c|}{ (Continuous recording) } \\
\hline Co-medication, (adjuvant) & \multicolumn{13}{|c|}{ (Continuous recording) } \\
\hline
\end{tabular}

\section{Follow-up}

Patients will be followed for up to 5 years after the intervention according to the visit schedule detailed in Table 1 . In each visit, a physical examination and recurrence assessment will be performed. An image assessment will be requested every year after the intervention. Reasons for discontinuing follow-up must be reported.

\section{Data collection and analysis}

Data collection

The study data will be recorded in an eCRF (OpenClinica ${ }^{\circledR}$, LLC). The demographic and clinical data requested is depicted in Table 2. Acute and chronic toxicity will be recorded according to the Common Terminology
Criteria for Adverse Events v4.0 (CTCAE) [24] criteria. Specific follow-up outcomes will be assessed and registered during the visits scheduled as stated in Table 1.

\section{Sample size estimation}

A total of 1.400 patients must be recruited to show the non-inferiority of the experimental arm (incidental irradiation) with an $80 \%$ of statistical power when we assume a 5 -year recurrence rate of $15 \%$ in the control arm (intentional irradiation) [8], a 5\% non-inferiority margin, a yearly dropout rate of $5 \%$, and a fixed sample design. Despite the short follow-up period, the large number of patients to be included in the study will preserve the

Table 2 Data collected in the electronic case record form

\begin{tabular}{ll}
\hline Panel in eCRF & Data recorded \\
\hline Informed consent & Date \\
I/E criteria & Yes; not \\
Demographic data & Age at inclusion; Menstrual State \\
Comorbidities & If yes: specify \\
Cancer Histology and Receptors, SLN OSNA & Tumour grade; Tumour size (maximum diameter); P53 (\%); Ki67 (\%); Lymphovascular infiltration; Ductal Ca \\
& in situ; \% Estrogenic receptors; \% Progesterone receptors; HER2 receptor status; OSNA TTL of SLN \\
Previous medication (Adjuvant therapy) & Drug; Start date; Stop date \\
Type of surgery & Tumour surgery; date; Margins \\
Randomization & Treatment randomly allocated; Randomization date \\
Radiotherapy intervention & Patient completed the allocated treatment (If no: main reason); Start date; End date; Treatment gaps (If yes, \\
& reason); Dose per volume (Mean; Median; D95; D5; Volume) in the breast, tumour bed, supraclavicular \\
Co-medication & and axillary levels I-III, and Internal mammary chain \\
Survival and disease recurrence & Drug; Start date; Stop date \\
& Date of follow-up visit (If not performed, reason); Local recurrence; Regional recurrence (If yes: nodal level); \\
Image evaluation of local recurrence & Distant recurrence (If yes: organ); Vital status (if dead: date and cause) \\
Physical examination ${ }^{2}$ & Technique; Local recurrence (if yes: Maximum diameter) \\
& Palpable breast tumour (if yes: size, skin infiltration, inflammatory carcinoma, satellite lesions); Palpable \\
Acute and chronic toxicity & axillary nodes (if yes: size); Palpable supraclavicular nodes (if yes: size); Node staging \\
\hline
\end{tabular}

eCRF electronic case record form, OSNA One-Step Nucleic Acid Amplification, TTL total tumour load, SLN sentinel lymph node, CTCAE Common Terminology Criteria for Adverse Events

a Physical examination is conducted at follow-up visits. It is also addressed at baseline and radiotherapy sessions to confirm the compliance of eligible criteria. If suspicious LNs are detected, the patient will discontinue the study 
statistical significance of the survival rates and will be enough to evaluate differences in the toxicity rates.

\section{Statistical analysis}

Two sets of patients will be analysed: the intentionto-treat (ITT) group, which includes all randomized patients, to describe the baseline clinic-pathological patients' characteristics; and the per-protocol subset, which includes patients who finish the intervention treatment as planned with all dosimetry data completed, for the endpoint. A descriptive analysis will be carried out reporting absolute and relative frequencies for all variables recorded and stratified by treatment group. Two analyses of the primary endpoint (disease-free survival rate) will be conducted. The confirmatory analyses will be carried out using a non-inferiority long-rank test in the ITT set of patients, which includes all randomized patients regardless of whether the treatment or followup are accomplished. A secondary explanatory analysis will be conducted in the per-protocol subgroup, which will include patients who will have finished the intervention treatment with all dosimetry data completed, by an adjusted Cox regression model using the covariates: centre, age at inclusion, tumour size, hormone receptor status, Her2 receptor status, and OSNA results. With regards to the secondary endpoints: the outcomes of loco-regional and distant recurrence will be analysed using the Cox approach, this time in the ITT set. Acute toxicity will be analysed by a chi-squared test comparing frequencies in both treatment groups. Chronic toxicity will be analysed by using Kaplan Meier curves and comparing them by a standard log-rank test. Interim analyses are planned when 85 events and 169 events will be reached.

\section{Current status of the trial}

From February 2015 to February 2020, a total of 451 patients have been recruited (224 in the intentional arm and 227 in the incidental arm). Currently, acute toxicity events have been reported in 319 cases. A total of 48 chronic toxicity and 13 recurrence events have been also informed.

\section{Discussion}

Nowadays, early BC patients with 2 or fewer involved LN undergo nodal irradiation instead of lymphadenectomy although it is unclear whether these patients require local-specific treatment. This uncertainty arises from that current studies do not clearly describe the radiation volumes used as adjuvant treatment. Furthermore, some physicians advocate that the breast of these patients should be treated with high or modified tangents [25]. Therefore, although it is not stated as LN irradiation, the axillary levels I and II are being irradiated. Accordingly, more than $70 \%$ of patients who had not undergone lymphadenectomy received $\mathrm{LN}$ radiation and even $19 \%$ received unallowed supraclavicular irradiation in the study conducted by Giuliano et al. [26]. In line with previous research, a recent larger meta-analysis reported that expanding the radiation field to the axillary and supraclavicular nodes after ALND reduces locoregional recurrences without an improvement on the overall and cancer-specific survival [27]. Thus, patients' survival would be improved due to the inclusion of the internal mammary chain in the radiation plan. Consequently, elucidating the effect of node radiation by means incidental or intentional doses in early $\mathrm{BC}$ is needed.

Thus, the OPTIMAL trial was designed to outline an evidence-based strategy for the treatment of $\mathrm{BC}$ patients with limited nodal involvement by determining the noninferiority of incidental radiation of the axillary nodes in comparison with intentional radiation. The ESTRO (European Society for Radiotherapy and Oncology) meeting in Assisi stated the importance of further investigations on regional lymph node treatment and highlighted the design and expected results of the OPTIMAL, SENOMAC, and POSNOC studies, all of them focused on the treatment of patients with limited axillary disease [28].

Final results from the INSEMA and SOUND trials $[29,30]$, which mainly questioned the use of no-ALND instead of SLN biopsy procedures in early BC, may also contribute to better outline treatment strategies in early BC. Nonetheless, even though patients with limited LN involvement (1-3 macrometastases) are considered in these studies, whole-breast radiation therapy is conducted.

In the OPTIMAL trial, the SLN status and the limited LN involvement is determined according to the OSNA quantitative molecular assay [20]. At the time of the study design, few studies with regards to OSNA performance had been reported. Nowadays, its contribution to the improvement of $\mathrm{BC}$ patient staging and prognosis has been widely reported [31]. In fact, LN assessment with OSNA assay and the evaluation of the TTL as a quantitative score of the metastatic burden of the patients is recommended for the management of $\mathrm{BC}$ patients in the SESPM (Spanish Society of Senology and Breast Pathology) and the NICE (National Institute for Health and Care Excellence) guidelines [32-34]. This technique provides quick and standardized results at in-house diagnostic laboratories, which prompts the patient's diagnosis and treatment tailoring since central laboratories are not required for a reliable $\mathrm{LN}$ assessment.

In conclusion, due to the lack of consensus on the proper therapeutic strategy of BC patients with few 
involved LNs standardizing the treatment and diagnosis is crucial. Hence, the quantitative score for metastatic burden provided by OSNA assay can contribute by improving the discrimination of the $\mathrm{BC}$ patients with a limited nodal involvement who can benefit from incidental radiation as an adjuvant treatment strategy.

\begin{abstract}
Abbreviations
ALND: Axillary lymph node dissection; AP/PA: Anterior-posterior/posterioranterior; BC: Breast cancer; CK19: Cytokeratin 19; CTCAE: Common Terminology Criteria for Adverse Events; DFS: Disease-free survival; eCRF: Electronic case report form; ESTRO: European Society for Radiotherapy and Oncology; ITT: Intention-to-treat; LN: Lymph node; mRNA: Messenger ribonucleic acid; NICE: National Institute for Health and Care Excellence; OSNA: One-Step Nucleic Acid Amplification; SESPM: Spanish Society of Senology and Breast Pathology; SLN: Sentinel lymph node; TTL: Total tumour load.
\end{abstract}

\section{Acknowledgements}

Thanks to all collaborators of the OPTIMAL study group and all patients who participated in the OPTIMAL study. Aurea Manso, Radiation Oncology, Hospital La Paz, Madrid; Andere Frías, Radiation Oncology, Cruces University Hospital, Bilbao; Ezequiel González, Radiation Oncology, University Clinical Hospital of Santiago de Compostela; Ana Calín, Radiation Oncology, University General Gregorio Marañón, Madrid; Inmaculada Díaz, Radiation Oncology, Oncology Centre of Galicia, A Coruña; Elena Guimón, Radiation Oncology, Donostia University Hospital; Antonio José Lozano, Radiation Oncology, University Clinical Hospital Virgen de la Arrixaca, Murcia; Raúl Herranz de Lucas, Radiation Oncology, Ramon y Cajal University Hospital, Madrid; María Luisa Lopez Louzara, Radiation Oncology, Meixoeiro Hospital, Vigo; Izaskun Valduvieco, Radiation Oncology, Clínic Hospital, Barcelona; Ana Manterola, Radiation Oncology, Navarra Hospital Complex, Pamplona; Pastora Caballero, Radiation Oncology, Fuenlabrada University Hospital, Madrid; Ignacio Andrés García, Radiation Oncology, Albacete University Hospital Complex; Francisco Carrasco, Radiation Oncology, Virgen Macarena University Hospital, Seville; Esther Jordà, Radiation Oncology, University of Valencia Clinical Hospital; Reyes Ibañez, Radiation Oncology, Miguel Servet University Hospital, Zaragoza; Pino Alcantara, Radiation Oncology, San Carlos Clinical Hospital, Madrid; Yesika Ríos, Radiation Oncology, Infanta Cristina Hospital, Badajoz; Amparo González, Radiation Oncology, University of Valencia General Hospital; Pedro Meireles, Radiation Oncology, São João Hospital Centre, Porto; Meritxell Arenas, Radiation Oncology, Sant Joan de Reus University Hospital; María Isabel Tortajada Azcutia, Radiation Oncology, Valencian Institute of Oncology, Valencia; Agustí Pedro, Radiation Oncology, Plató Hospital, Barcelona; Miguel Manuel Sanz Martín, Radiation Oncology, General University Hospital of Ciudad Real; Carmen Velilla, Radiation Oncology, Lozano Blesa University Clinical Hospital, Zaragoza; Laura García, Radiation Oncology, Doctor Negrin University Hospital of Gran Canaria, Gran Canaria.

\section{Authors' contributions}

Study design, manuscript conception and writing: MA and XS. Every author reviewed the manuscript and substantially contributed to the current patient recruitment. All authors read and approved the final manuscript.

\section{Funding}

The sponsor GICOR (Grupo de Investigación Clínica en Oncología Radioterápica), a non-profit research organization, is responsible for ensuring compliance with legal regulations and study management. GICOR has the final responsibility for publication. A Clinical Research Organization (Saalig Clinical, S.L.) supports the Sponsor on study monitoring, data collection and analysis. Sysmex Europe provides technical and financial support for IRB fees and eCRF maintenance. The corporation was not involved in the study design, data collection nor analysis.

\section{Availability of data and materials}

The datasets generated and/or analysed during the current study are not publicly available due to confidentiality reasons but are available from the corresponding author on reasonable request.

\section{Ethics approval and consent to participate}

The study will be conducted in accordance with the Declaration of Helsinki-Ethical Principles for Medical Research Involving of Human Subjects and guidelines for Good Clinical Practice. The Spanish Agency of Medicines and Medical Devices evaluated and classified the study. The study has been approved by the Institution Research Board of each centre.

\section{Consent for publication}

Not applicable.

\section{Competing interests}

Dr. Algara has received consulting honoraria from Sysmex and Aristo and speaking honoraria from Siemens and Roche; the rest of authors declare that they have no competing interests.

\section{Author details}

${ }^{1}$ Radiation Oncology Department, Del Mar Hospital, Autonomous University of Barcelona, Hospital del Mar Medical Research Institute, Passeig Maritim, 25, 08003 Barcelona, Spain. ${ }^{2}$ Radiation Oncology Department, Virgen del Rocío University Hospital, Seville, Spain. ${ }^{3}$ Radiation Oncology Department, Castellón Provincial Hospital, Castellón de La Plana, Spain. ${ }^{4}$ Radiation Oncology Department, La Fe Polytechnic University Hospital La Fe, Valencia, Spain. ${ }^{5}$ Radiation Oncology Department, Santa Lucia General University Hospital, Cartagena, Spain. ${ }^{6}$ Radiation Oncology Department, University Hospital of Leon, León, Spain. ${ }^{7}$ Radiation Oncology Department, Del Mar Hospital, Pompeu Fabra University, Hospital del Mar Medical Research Institute, Barcelona, Spain.

${ }^{8}$ Radiation Oncology Department, De La Ribera Hospital, Alzira, Spain.

${ }^{9}$ Radiation Oncology Department, Cabueñes University Hospital, Gijón, Spain.

${ }^{10}$ Araba Txagorritxu University Hospital, Vitoria, Spain.

Received: 24 April 2020 Accepted: 24 September 2020

Published online: 02 October 2020

\section{References}

1. Abe O, Abe R, Enomoto K, Kikuchi K, Koyama H, Masuda H, et al. Effects of radiotherapy and of differences in the extent of surgery for early breast cancer on local recurrence and 15-year survival: an overview of the randomised trials. Lancet. 2005;366:2087-106.

2. Recht A, Comen EA, Fine RE, Fleming GF, Hardenbergh PH, Ho AY, et al. Postmastectomy radiotherapy: an american society of clinical oncology, american society for radiation oncology, and society of surgical oncology focused guideline update. Pract Radiat Oncol. 2016;6:e219-34.

3. Dalela D, Santiago-Jiménez M, Yousefi K, Karnes RJ, Ross AE, Den RB, et al. Genomic classifier augments the role of pathological features in identifying optimal candidates for adjuvant radiation therapy in patients with prostate cancer: development and internal validation of a multivariable prognostic model. J Clin Oncol. 2017;35:1982-90.

4. Ragaz J, Olivotto IA, Spinelli JJ, Phillips N, Jackson SM, Wilson KS, et al. Locoregional radiation therapy in patients with high-risk breast cancer receiving adjuvant chemotherapy: 20-year results of the British Columbia randomized trial. J Natl Cancer Inst. 2005;97:116-26.

5. Overgaard M, Nielsen HM, Overgaard J. Is the benefit of postmastectomy irradiation limited to patients with four or more positive nodes, as recommended in international consensus reports? A subgroup analysis of the DBCG 82 b\&c randomized trials. Radiother Oncol. 2007;82:247-53.

6. Whelan TJ, Olivotto IA, Parulekar WR, Ackerman I, Chua BH, Nabid A, et al. Regional nodal irradiation in early-stage breast cancer. N Engl J Med. 2015;373:307-16

7. Xie L, Higginson DS, Marks LB. Elective regional nodal irradiation in patients with early-stage breast cancer. Semin Radiat Oncol. 2011;21:66-78.

8. Giuliano AE, Hunt KK, Ballman KV, Beitsch PD, Whitworth PW, Blumencranz PW, et al. Axillary dissection vs no axillary dissection in women with invasive breast cancer and sentinel node metastasis. JAMA. 2011;305:569-75

9. Giuliano AE, McCall L, Beitsch P, Whitworth PW, Blumencranz P, Leitch $\mathrm{AM}$, et al. Locoregional Recurrence after Sentinel Lymph Node Dissection with or without Axillary Dissection in Patients with Sentinel Lymph Node 
Metastases: the American College of Surgeons Oncology Group Z0011 Randomized Trial. Ann Surg. 2010;252:426-33.

10. Straver ME, Meijnen P, Van Tienhoven G, Van De Velde CJH, Mansel RE, Bogaerts J, et al. Sentinel node identification rate and nodal involvement in the EORTC 10981-22023 AMAROS trial. Ann Surg Oncol. 2010;17:1854-61.

11. Sávolt A, Péley G, Polgár C, Udvarhelyi N, Rubovszky G, Kovács E, et al. Eight-year follow up result of the OTOASOR trial: The Optimal Treatment Of the Axilla — Surgery Or Radiotherapy after positive sentinel lymph node biopsy in early-stage breast cancer: a randomized, single centre, phase III, non-inferiority trial. Eur J Surg Oncol. 2017;43:672-9.

12. Poortmans PM, Collette S, Kirkove C, Van Limbergen E, Budach V, Struikmans $\mathrm{H}$, et al. Internal mammary and medial supraclavicular irradiation in breast cancer. N Engl J Med. 2015;373:317-27.

13. Darby S, McGale P, Correa C, Taylor C, Arriagada R, Clarke M, et al. Effect of radiotherapy after breast-conserving surgery on 10-year recurrence and 15-year breast cancer death: Meta-analysis of individual patient data for 10801 women in 17 randomised trials. Lancet. 2011;378:1707-16.

14. Van Wely BJ, Teerenstra S, Schinagl DAX, Aufenacker TJ, De Wilt JHW, Strobbe LJA. Systematic review of the effect of external beam radiation therapy to the breast on axillary recurrence after negative sentinel lymph node biopsy. Br J Surg. 2011;98:326-33.

15. Budach W, Kammers K, Boelke E, Matuschek C. Adjuvant radiotherapy of regional lymph nodes in breast cancer-a meta-analysis of randomized trials. Radiat Oncol. 2013;8:1-7.

16. Budach W, Bölke E, Kammers K, Gerber PA, Nestle-Krämling C, Matuschek C. Adjuvant radiation therapy of regional lymph nodes in breast cancer - a meta-analysis of randomized trials - an update. Radiat Oncol. 2015;10:1-7. https://doi.org/10.1186/s13014-015-0568-4.

17. Setton J, Cody H, Tan L, Morrow M, Hudis C, Catalano J, et al. Radiation field design and regional control in sentinel lymph node-positive breast cancer patients with omission of axillary dissection. Cancer. 2012;118:1994-2003.

18. Haffty BGB, Hunt KKK, Harris JRJ, Buchholz TA. Positive sentinel nodes without axillary dissection-implications for the radiation oncologist. J Clin Oncol. 2011;29:4479-81.

19. Bayo E, Herruzo I, Arenas M, Algara M. Consensus on the regional lymph nodes irradiation in breast cancer. Clin Transl Oncol. 2013;15:766-73.

20. Tsujimoto M, Nakabayashi K, Yoshidome K, Kaneko T, Iwase T, Akiyama $F$, et al. One-step nucleic acid amplification for intraoperative detection of lymph node metastasis in breast cancer patients. Clin Cancer Res. 2007;13:4807-16

21. Peg V, Espinosa-Bravo M, Vieites B, Vilardell F, Antúnez JR, De Salas MS, et al. Intraoperative molecular analysis of total tumor load in sentinel lymph node: A new predictor of axillary status in early breast cancer patients. Breast Cancer Res Treat. 2013;139:87-93.

22. Peg V, Sansano I, Vieites B, Bernet L, Cano R, Córdoba A, et al. Role of total tumour load of sentinel lymph node on survival in early breast cancer patients. The Breast. 2017;33:8-13.

23. Li XA, Ph D, Tai A, Ph D, Arthur DW, Buchholz TA, et al. Variability of target and normal structure delineation for breast cancer radiotherapy: an
RTOG Multi-Institutional and Multiobserver Study [Breast Cancer Atlas for Radiation Therapy Planning: Consensus Definitions]. Int J Radiat Oncol Biol Phys. 2009;73:944-51.

24. National Cancer Institute. Common Terminology Criteria for Adverse Events (CTCAE) v4.0. 2010. https://ctep.cancer.gov/protocolDevelop ment/electronic_applications/ctc.htm\#ctc_40

25. Gebhardt BJ, Thomas J, Horne ZD, Champ CE, Ahrendt GM, Diego E, et al. Standardization of nodal radiation therapy through changes to a breast cancer clinical pathway throughout a large, integrated cancer center network. Pract Radiat Oncol. 2018;8:4-12.

26. Jagsi R, Chadha M, Moni J, Ballman K, Laurie F, BuchholzTA, et al. Radiation field design in the ACOSOG Z0011 (Alliance) trial. J Clin Oncol. 2014;32:3600-6.

27. Haussmann J, Budach W, Tamaskovics B, Bölke E, Corradini S, DjiepmoNjanang $F$, et al. Which target volume should be considered when irradiating the regional nodes in breast cancer? Results of a network-metaanalysis. Radiat Oncol. 2019;14:1-14.

28. Aristei C, Kaidar-Person O, Arenas M, Coles C, Offersen BV, Bourgier C, et al. The 2016 Assisi Think Tank Meeting on breast cancer: white paper. Breast Cancer Res Treat. 2016;160:211-21.

29. Reimer T, Stachs A, Nekljudova V, Loibl S, Hartmann S, Wolter K, et al. Restricted axillary staging in clinically and sonographically node-negative early invasive breast cancer (C/IT1-2) in the context of breast conserving therapy: first results following commencement of the intergroup-sentinel-mamma (INSEMA) trial. Geburtshilfe Frauenheilkd. 2017;77:149-57.

30. Gentilini $\mathrm{O}$, Veronesi U. Abandoning sentinel lymph node biopsy in early breast cancer? A new trial in progress at the European Institute of Oncology of Milan (SOUND: Sentinel node vs Observation after axillary UltraSouND). Breast. 2012;21:678-81. https://doi.org/10.1016/j.breas t.2012.06.013.

31. Chaudhry A, Williams S, Cook J, Jenkins M, Sohail M, Calder C, et al. The real-time intra-operative evaluation of sentinel lymph nodes in breast cancer patients using One Step Nucleic Acid Amplification (OSNA) and implications for clinical decision-making. Eur J Cancer Surg . 2014:40:150-7.

32. Bernet L, Piñero A, Vidal-Sicart S, Peg V, Giménez J, Algara M, et al. Consenso sobre la biopsia selectiva del ganglio centinela en el cáncer de mama. Revisión 2013 de la Sociedad Española de Senología y Patología Mamaria. Rev Esp Patol. 2014;47:22-32.

33. National Institute for Health and Clinical Excellence. Intraoperative tests (RD-100i OSNA system and Metasin test) for detecting sentinel lymph node metastases in breast cancer. Diagnostics guidance [DG8]; 2013. https://www.nice.org.uk/nicemedia/live/14247/64810/64810.pdf

34. Szabatura AH, Pharm D, Seung AH, Pharm D, Freml JM, Pharm D, et al. Early and locally advanced breast cancer. Natl Inst Heal Clin Excell. 2019;56:1-32.

\section{Publisher's Note}

Springer Nature remains neutral with regard to jurisdictional claims in published maps and institutional affiliations.

\footnotetext{
Ready to submit your research? Choose BMC and benefit from:

- fast, convenient online submission

- thorough peer review by experienced researchers in your field

- rapid publication on acceptance

- support for research data, including large and complex data types

- gold Open Access which fosters wider collaboration and increased citations

- maximum visibility for your research: over 100M website views per year
}

At $\mathrm{BMC}$, research is always in progress.

Learn more biomedcentral.com/submissions 